\title{
Relación entre la cohesión grupal y la ansiedad competitiva en fútbol de iniciación.
}

\section{Relationship between group cohesion and competitive anxiety in initiation football}

Fecha de recepción: 16-02-2021

Fecha de aceptación: 18 -07-2021

\author{
Julia Pérez Cáceres \\ Universidad de Granada. España \\ M Rocio Bohórquez Gómez-Millán \\ Universidad de Sevilla. España \\ M Ángeles Arias Velarde \\ Universidad de Sevilla. España
}

\section{resumen/alsstract:}

El presente estudio tiene como objetivo principal analizar la relación entre la cohesión grupal y la ansiedad competitiva en jugadores de fútbol de iniciación. La literatura ha demostrado que los factores de tarea de la cohesión grupal se relacionan de forma inversa con la ansiedad competitiva, aunque este aspecto no ha sido explorado en deporte infantil. En el estudio participaron 60 jugadores de fútbol pertenecientes a distintas categorías de un club deportivo y con una edad media de 12.32 años (DT = 1.73). Los resultados revelaron que aquellos jugadores que puntuaban alto en integración grupal en la tarea mostraban menos preocupación acerca de la competición, del mismo modo, jugadores con elevada atracción grupal hacia lo social mostraron menor preocupación sobre la competición. Se discuten estos resultados en relación con la literatura previa, encontrado diferencias con el funcionamiento de la relación cohesión-ansiedad en población de deportistas jóvenes y adultos profesionales y semiprofesionales.

The present study's main objective was to analyze the relationship between group cohesion and competitive anxiety in initiation soccer players. The literature has shown that the task factors of group cohesion are inversely related to competitive anxiety, although this aspect has not been explored in children's sports. 60 soccer players participated in the study belonging to different categories of a sports club and with a mean age of 12.32 years (DT $=1.73$ ). The results revealed that those players who scored high in group integration in the task showed less concern about the competition, in the same way, players with high group social attraction showed less concern about the competition. These results are discussed concerning to the previous literature, finding differences with the functioning of the cohesion-anxiety relationship in the population of young athletes and professional and semi-professional adults.

\section{palahras clave/keywords:}

cohesión grupal, ansiedad competitiva, deporte infantil, iniciación deportiva, fútbol.

group cohesion, competitive anxiety, children's sport, soccer, 
La cohesión grupal es considerada como la variable más importante de los grupos (Golembiewski, 1962; Lott y Lott, 1965) y se trata de un concepto clave en la formación y desarrollo de los equipos de trabajo (Barrasa y Gil, 2004), por lo que su estudio despierta un gran interés en los ámbitos de la psicología social y del deporte (Coleman et al, 2021; ChicauBorrego et al, 2021). La definición de cohesión grupal más utilizada la aportó Carron (1982) quien la describe como "el proceso dinámico que se refleja en la tendencia de un grupo de mantenerse juntos y permanecer unidos en la persecución de sus metas y objetivos" ( $p$. 124). Esta definición se basa en un modelo multifactorial que concibe la cohesión como un constructo multidimensional y dinámico; por una parte, los miembros tienen percepciones acerca del grupo, surgiendo así las dimensiones de integración grupal (que se refiere al grado en el que el grupo actúa de forma conjunta o como un todo), y de atracción hacia el grupo, que se entiende como el grado en el que el grupo satisface las necesidades individuales de cada miembro (Tenebaum et al., 2012). Por otra parte, en función del enfoque, surgen la dimensión interpersonal o de enfoque social, refiriéndose al grado de compañerismo y empatía existente entre los miembros, y la dimensión instrumental o enfoque hacia la tarea, haciendo referencia al grado en el que el grupo trabaja unido para conseguir objetivos comunes (Carron et al., 1985; 1998). A partir de estas dimensiones surgen cuatro factores principales de los que se compone el constructo de la cohesión grupal (Carron, 1982; Carron et al, 2007): integración en el componente social (IG-S), integración en la tarea grupal (IG-T), atracción individual hacia lo social (AIG-S) y atracción individual hacia la tarea (AIG-T).

El interés por el estudio de la cohesión grupal dentro de la psicología del deporte proviene tanto de los factores que anteceden a la misma, por ejemplo, factores ambientales, personales, de liderazgo o de equipo (Bohórquez et al., 2012; 2017), como del efecto que esta ejerce sobre otros procesos grupales como la comunicación y el rendimiento deportivo (Eys et al., 2003; Prapavessis y Carron, 1996; Terry et al., 2000; Williams, 1991). Sin embargo, uno de los temas que ha despertado mayor interés ha sido el estudio de la influencia de la cohesión grupal sobre ciertas variables psicológicas adversas como es el caso de la ansiedad competitiva (Borrego et al., 2012; Oh, 2014; Prapavessis y Carron, 1996; Wolf et al., 2015), la cual deriva, en gran parte, de los procesos de estrés desencadenados por las percepciones sobre la importancia de la competición y la incertidumbre sobre el resultado (Burton, 1998).

Morris et al. (1981) define la ansiedad competitiva como "las expectativas negativas y las preocupaciones cognitivas sobre uno mismo, la situación en cuestión y las posibles consecuencias" (p.74). Además, ésta es entendida como un constructo que comprende dos grandes dimensiones: la ansiedad somática y la ansiedad cognitiva. La ansiedad somática se refiere a los elementos psicológicos y afectivos de la experiencia de la ansiedad, que se desarrollan directamente a partir del propio nivel de activación, mientras que la ansiedad cognitiva es considerada el componente mental de la ansiedad, causada por las expectativas negativas ante una situación o una autoevaluación (Martens et al., 1990). Algunos autores proponen que la dimensión cognitiva de la ansiedad puede dividirse, a su vez, en dos niveles: preocupación, que se refiere a la inquietud por las posibles consecuencias negativas por un rendimiento pobre, y desconcentración, entendida como la dificultad del deportista para focalizarse en los aspectos clave de la tarea, impidiendo un pensamiento claro durante la situación competitiva (Grossbard et al., 2009). 
Diferentes autores han demostrado que, tanto la intensidad como la percepción (positiva o negativa) de la ansiedad competitiva están estrechamente relacionadas con el desempeño deportivo (Eys y Kim, 2021; Neil et al., 2012). Por este motivo, muchas investigaciones se han interesado por el estudio de la interacción de la ansiedad competitiva con otras variables como la cohesión grupal. Se ha postulado que la cohesión enfocada a la tarea correlaciona inversamente con el estado de ansiedad competitiva (Prapavessis \& Carron, 1996; Borrego et al., 2012). Concretamente, se observó que la atracción individual hacia el grupo se correlacionaba con una disminución de la ansiedad cognitiva, mientras que la integración grupal se relacionaba con valores más bajos de ansiedad tanto cognitiva como somática (Borrego et al., 2012). En estudios en los que se evalúo tanto el enfoque instrumental como el social hallaron resultados similares, concluyendo que aquellos grupos que presentaban mayores niveles de cohesión grupal, tanto social como instrumental, mostraban percepciones más bajas de ansiedad competitiva (Wolf et al., 2015). Cabe destacar que este estudio, a diferencia de los mencionados anteriormente, además de evaluar la intensidad de la ansiedad vivida, también exploró la percepción, positiva o negativa, de la misma. Finalmente, observaron que la obtención de valores altos de cohesión grupal predecía una interpretación de la ansiedad mas positiva o facilitadora (Wolf et al., 2015). Otras investigaciones han estudiado el efecto modulador de la cohesión sobre la relación entre la ansiedad competitiva y otras variables como el bienestar subjetivo del grupo, concluyendo que mayores niveles de cohesión grupal se relacionan con una debilitación del efecto negativo de la ansiedad sobre el bienestar psicológico de los jugadores (Aguinaga et al., 2020).

Por otra parte, aunque las investigaciones comentadas hasta ahora se han centrado en la influencia directa o indirecta de la cohesión sobre la ansiedad, hay estudios que han demostrado que la interacción entre la cohesión grupal y el estado de ansiedad competitiva no es unidireccional, sino que, más bien se trata de una relación dinámica en la que ambas variables pueden influirse mutuamente (Eys et al., 2003). Esta afirmación es apoyada por diversos estudios, tanto enfocados a la percepción de la ansiedad, en los que se ha observado que los atletas con una percepción facilitadora de su ansiedad competitiva obtienen valores más altos de cohesión que aquellos con una percepción negativa de su ansiedad (Eys et al., 2003), como centrados en la intensidad de la ansiedad vivida, en los que se ha observado que el aumento del nivel de ansiedad competitiva se relaciona con una mayor unión del equipo y, por tanto, con un mayor nivel de cohesión (Nascimento Junior et al., 2016).

Finalmente, cabe destacar que la mayoría de las investigaciones llevadas a cabo sobre la relación entre la cohesión grupal y el estado de ansiedad competitiva han sido desarrolladas en el ámbito del deporte profesional, excepto un trabajo llevado a cabo por Oh y Gill (2014) en el que se evalúo a 114 futbolistas adultos amateur. Los resultados revelaron que todos los factores de la cohesión, excepto la atracción individual hacia lo social, obtuvieron una correlación negativa con respecto al estado de ansiedad competitiva, tanto cognitiva como somática (Oh y Gill, 2014). Sin embargo, se desconocen estudios que exploren esta cuestión en el de futbol de iniciación, en los que los evaluados sean futbolistas jóvenes.

Por todo ello, el presente estudio se plantea como principal objetivo examinar la relación entre todos los factores de la cohesión grupal (AIG-S, AIG-T, IG-S e IG-T) y las diferentes 
dimensiones del estado de ansiedad competitiva (somática, preocupación y desconcentración) en un grupo de futbolistas jóvenes no profesionales. Este objetivo principal se desglosa en cuatro hipótesis de investigación: (1) el factor integración del grupo - tarea (IGT) influirá de forma inversa sobre las tres dimensiones de la ansiedad competitiva; (2) el factor atracción individual al grupo - tarea (AIG-T) influirá de forma inversa sobre las tres dimensiones de la ansiedad competitiva; (3) el factor integración del grupo - social (IG-S) no correlacionará con ninguna de las dimensiones de la ansiedad competitiva y (4) el factor atracción individual al grupo - social (AIG-S) no correlacionará con ninguna de las dimensiones de la ansiedad competitiva.

\section{Método}

\section{Participantes}

Participaron 60 niños de sexo masculino miembros de un club deportivo de fútbol de Sevilla. Los jugadores competían en las categorías Alevín de $1^{\circ}$ año, alevín de $2^{\circ}$ año, Infantil y Cadete. Los participantes cursaban entre $4^{\circ}$ curso de primaria y $4^{\circ}$ de la E.S.O., con una edad media de 12.32 años $(\mathrm{DT}=1,73)$ en un rango comprendido entre los 9 y los 16 años.

La muestra se constituyó mediante por muestreo intencional no probabilístico por accesibilidad al club deportivo. Dado que al inicio de temporada los tutores legales de los jugadores firmaron un consentimiento informado en el que se incluía la autorización para participar en procesos de investigación no siendo, por consiguiente, necesaria la solicitud de un nuevo permiso para la colaboración de los participantes en el presente estudio, que accedieron a intervenir de forma voluntaria, habiendo sido previamente informados tanto de los objetivos perseguidos como de la no obligatoriedad de su participación.

\section{Instrumentos}

Cuestionario de datos sociodemográficos. Se utilizó un cuestionario creado ex profeso para recoger información acerca de la edad, el curso escolar, el tiempo en el club, la categoría, la posición en el campo y la preferencia por competir (sí o no) de los participantes.

Cohesión grupal. Para medir la cohesión grupal se utilizó la adaptación española llevada a cabo por Leo et al. (2015) de la herramienta Group Environment Questionaire (CEG; Carron et al., 1985), la cual fue validada con una alta consistencia interna de $\alpha>.70$ para la escala general y valores de .60 para AIG-S, .77 para AIG-S, .76 para el factor IG-S y .74 para IG-T; e índices aceptables en su estructura factorial $(\chi 2 / d f=4.39$; $\mathrm{CFI}=.95)$ (Leo et al., 2015). Se trata de un cuestionario formado por 12 ítems agrupados en cuatro factores: integración grupo-tarea (IG-T), por ejemplo, “los miembros del equipo unen sus esfuerzos para conseguir los objetivos durante los entrenamientos y los partidos", al que corresponden los ítems 8, 10 y 12; integración grupo-social (IG-S), relacionado con el ítem 7 " a los miembros del equipo les gusta quedar juntos" , y los ítems 9 y 11; atracción individual hacia el grupo-tarea (AIG-T), al que pertenecen los ítems 2 "estoy contento con mi aportación al equipo”, 4 y 6; y, por último, atracción individual hacia el grupo-social (AIG-S), a los que corresponden los ítems 3 "tengo buenos amigos en este equipo", 1 y 5. La escala tuvo una fiabilidad en nuestra muestra de .713, y un RMSEA de .042. 
En el cuestionario original, cada ítem se mide con una escala de tipo Likert de 9 puntos, en la que 1 equivaldría a "totalmente en desacuerdo" y 9 "totalmente de acuerdo". Sin embargo, para la presente investigación, se decidió reducir la escala a 5 puntos, ya que algunos estudios han reflejado que, al tratarse de niños, esta reducción podría facilitar la recogida de datos en esta población y disminuir los riesgos de polarización de las respuestas (Alan y Kabasakal, 2020; Chambers y Johnston, 2002).

Ansiedad Competitiva. Para recoger los datos sobre la ansiedad, se utilizó el Cuestionario de Ansiedad Competitiva SAS-2 (Ramis et al., 2010) que se trata de una adaptación al español del original en inglés Sport Anxiety Scale-2 (Smith et al., 2006). Dicha adaptación se consideró válida obteniendo un coeficiente de alfa de Cronbanch de 0,83 para la subescala somática, de 0,78 para la subescala de preocupación y de 0,73 para la subescala de desconcentración (Ramis et al., 2010). El cuestionario se compone de 15 ítems que se dividen en tres factores: ansiedad somática, por ejemplo, ' '... siento que mi cuerpo est tenso' preocupación '... me preocupa no jugar o competir bien”; y desconcentración “...pierdo la concentración en el partido o la competición”. Estos ítems son medidos a través de una escala tipo Likert de 4 puntos comprendida entre 1 (nada) y 4 (mucho). Las puntuaciones totales por subescalas se obtienen a partir de la suma de las puntuaciones de sus ítems y pueden oscilar entre los 5 y 20 puntos. La escala tuvo una fiabilidad en nuestra muestra de .675 , y un RMSEA de .04.

\section{Procedimiento}

Se solicitó la cooperación del club a partir de un contacto con el presidente de éste. Una vez aprobada la colaboración, se informó a los entrenadores de cada equipo acerca de los objetivos y procedimientos del estudio y se fijó una fecha de evaluación. Siguiendo un protocolo de evaluación similar en todos los grupos, la administración se llevó a cabo en el mismo terreno de juego, antes de que los jugadores comenzaran el entrenamiento. Primero se les proporcionó información a los jugadores acerca de los distintos cuestionarios, explicando detalladamente las instrucciones para implementar cada uno de ellos. Se indicó a los jugadores que no había opciones correctas o incorrectas, se solicitó que contestaran con sinceridad y se aclaró que la participación en el estudio era voluntaria y la información recogida en el mismo sería anónima. A continuación, se llevó a cabo la administración del cuestionario sobre los datos sociodemográficos, seguido de la herramienta de cohesión grupal GEQ y, por último, el cuestionario sobre ansiedad SAS-2 bajo la supervisión de un único profesional que se encargó de evaluar todos los grupos. Los participantes consumieron un tiempo medio total de 20 minutos para completar la evaluación.

\section{Análisis de datos}

En primer lugar, se realizó un análisis descriptivo donde se muestran las puntuaciones medias obtenidas en las variables evaluadas, así como el mínimo, el máximo y la desviación estándar de cada factor. A continuación, se llevó a cabo un análisis de correlación bivariada de Pearson con el fin de determinar la correlación existente entre los factores de la cohesión grupal y las dimensiones de la ansiedad competitiva. Por último, se llevó a cabo un análisis de regresión múltiple para comprobar en qué medida los factores de la cohesión predecían 
cada componente del SAS-2. Se trabajó con un nivel de significación de $\mathrm{p}<.05$. Además, se calculó el tamaño de efecto con el índice , y la potencia de cada análisis, la cual se calculó a través del programa estadístico GPower 3 (Faul et al., 2009). A excepción de la potencia, los análisis se llevaron a cabo a través del programa SPSS (IBM, 2017).

\section{Resultados}

Los datos de ansiedad competitiva permiten observar que los sujetos obtienen una mayor puntuación media en el factor preocupación a diferencia de las restantes dimensiones, en las que se obtiene una puntuación media más baja (más cercana al mínimo) y, además, muy similar entre sí (Tabla 1). En cuanto a la cohesión, los resultados muestran, en general, niveles altos siendo la puntuación media muy parecida entre todos los factores que componen la escala (Tabla 2).

Tabla 1.- Resultados descriptivos de las dimensiones somática, preocupación y desconcentración de la ansiedad competitiva.

\begin{tabular}{cccccc} 
& $\boldsymbol{N}$ & Mínimo & Máximo & Media & DT \\
\hline EA- So & 60 & 5,00 & 18,00 & 8,233 & 3,072 \\
EA- P & 60 & 7,00 & 20,00 & 14,450 & 3,432 \\
EA- D & 60 & 5,00 & 19,00 & 9,317 & 3,619 \\
\hline
\end{tabular}

Nota. $\mathrm{N}=$ número de sujetos; DT= Desviación típica; EA-So= estado de ansiedadsomática; EA-P = Estado de ansiedad- preocupación; EA-D = Estado de ansiedad desconcentración.

Tabla 2.- Resultados descriptivos de los factores de la cohesión grupal.

\begin{tabular}{cccccc}
\hline & $\boldsymbol{N}$ & Mínimo & Máximo & Media & DT \\
\hline & & & & & \\
IG-T & 60 & 5,00 & 15,00 & 11,817 & 2,697 \\
IG-S & 60 & 4,00 & 15,00 & 11,733 & 2,705 \\
AIG-T & 60 & 6,00 & 15,00 & 12,900 & 2,305 \\
AIG-S & 60 & 5,00 & 15,00 & 12,733 & 2,530 \\
\hline
\end{tabular}

Nota. $\mathrm{N}=$ número de sujetos; DT= Desviación típica; IG-T = integración del grupo- tarea, IG-S = Integración grupo social; AIG-T = atracción individual hacia el grupo - tarea; AIG-S = atracción individual hacia el grupo - social. 
Se llevó a cabo un análisis de correlación bivariada de Pearson de todos los factores de la cohesión y las tres dimensiones de la ansiedad. En dicho análisis se observa que existe correlación inversa entre el factor IG-T y el estado de ansiedad competitiva, sin embargo, esta solo es significativa para la dimensión preocupación del estado de ansiedad competitiva $\left(\mathrm{R}^{\wedge} 2=.34 ; p=.007\right)$. Además, también puede observarse la existencia de una correlación inversa y significativa entre el factor AIG-S y la preocupación $(r=-.30 ; p=.021)$. Por otra parte, no se encontraron correlaciones significativas entre los restantes factores de la cohesión y las dimensiones de la ansiedad (Tabla 3).

Tabla 3.- Resultados de los análisis de correlación entre los factores de la cohesión grupal y las dimensiones del estado de ansiedad competitiva.

\begin{tabular}{|c|c|c|c|c|c|c|c|}
\hline & IG-T & IG-S & AIG-T & AIG-S & EA-So & EA-P & EA-D \\
\hline IG-T & 1 & $.36^{* *}$ & $.47 * *$ & $.26 * *$ & -.05 & $-.34 * *$ & -.20 \\
\hline IG-S & & 1 & .15 & .06 & .04 & -.10 & .01 \\
\hline AIG-T & & & 1 & $.39 * *$ & .03 & -.15 & .00 \\
\hline AIG-S & & & & 1 & .23 & $-.30 *$ & .05 \\
\hline EA-So & & & & & 1 & .136 & $.51 * *$ \\
\hline EA-P & & & & & & 1 & $.29 *$ \\
\hline EA-D & & & & & & & 1 \\
\hline
\end{tabular}

Nota. IG-T = integración del grupo- tarea, IG-S = Integración grupo social; AIG-T = atracción individual hacia el grupo - tarea; AIG-S = atracción individual hacia el grupo social; EA-So = estado de ansiedad- somática; EA-P = Estado de ansiedad- preocupación; EA-D $=$ Estado de ansiedad - desconcentración; $* \mathrm{p}<.05 . * * \mathrm{p}<.01$

En base a los resultados obtenidos, se llevó a cabo una regresión múltiple de aquellas variables relevantes, resultando así un modelo con los dos factores de la cohesión, IG-T y AIG$\mathrm{S}$, como variables predictivas y la dimensión preocupación de la ansiedad como variable dependiente $\left(\mathrm{R}^{\wedge} 2=.17, \mathrm{~F}(2,57)=5.64, p=.006\right)$. En dicha regresión, se encontró una influencia inversa y significativa de la variable IG-T sobre la preocupación $(\beta=-.29, t(59)$ $=-2.28, p=.026$ ) (Tabla 4). Es decir, aquellos jugadores con mayores valores de integración del grupo - tarea puntuaban valores menores en la dimensión preocupación de la ansiedad. Sin embargo, el factor AIG-T no mostró una relación significativa con la preocupación en 
presencia de la variable IG-S, es decir, la variable IG-T tiene una contribución única para la predicción de la preocupación. Estos resultados fueron concluidos con un tamaño de efecto alto $(=.17)$ y una potencia estadística de $1-\beta=.86$.

Tabla 4.- Coeficientes tipificados, t, y significación para las variables predictoras IG-T y AIG-S del modelo sobre la EA-P.

\begin{tabular}{lcccc}
\hline & $\beta$ & $\boldsymbol{t}$ & $\boldsymbol{g l}$ & $\boldsymbol{p}$ \\
\hline & & & & \\
IG-T & -.29 & -2.28 & 2 & .026 \\
AIG-S & -.22 & -1.78 & 2 & .080 \\
\hline
\end{tabular}

Nota. $g l=$ grados de libertad; $p=$ valores de significación; IG-T= integración del grupo tarea; AIG-S = atracción individual hacia el grupo - social.

\section{Discusión}

El objetivo principal del presente estudio era explorar la relación entre los diferentes factores de la cohesión grupal y las dimensiones del estado de ansiedad competitiva en deportistas jóvenes que practican fútbol de iniciación. En los resultados descritos se ha observado la existencia de una correlación negativa entre la percepción de cohesión grupal y el estado de ansiedad competitiva de los jugadores. Concretamente, se han hallado relaciones significativas entre los factores de IG-T y AIG-S de la cohesión y la dimensión preocupación de la ansiedad competitiva.

En relación con la dimensión instrumental de la cohesión, la primera hipótesis planteada defendía la existencia de una correlación inversa entre la (IG-T) y las diferentes dimensiones del estado de ansiedad competitiva. Dicha hipótesis se cumple para la relación entre IG-T y una de las dimensiones de la ansiedad: la preocupación. Además, los análisis de regresión múltiple muestran que el factor IG-T es el único factor de la cohesión que tiene un poder predictivo significativo sobre la preocupación. Sin embargo, aunque también se ha hallado una correlación entre esta dimensión de la ansiedad y AIG-T, esta no resulta significativa, rechazándose así la segunda hipótesis de la investigación. Las observaciones descritas son congruentes con las halladas en el estudio llevado a cabo por Wolf et al. (2015), aunque para estos autores con deportistas adultos. Otros trabajos (Borrego et al., 2012; Oh, 2014) coinciden con la correlación inversa hallada entre la IG-T y la ansiedad cognitiva, sin embargo, a diferencia del presente, estos estudios también observaron la existencia de una relación significativa con respecto al factor AIG-T y el estado de ansiedad, observando, de hecho, un mayor poder predictivo por parte de este factor. El hecho de que esta variable no tenga poder predictivo sobre la ansiedad puede deberse a que, en este estudio, el factor AIG-T se encuentra bastante correlacionado con el factor IG-T y muy poco con respecto a las dimensiones de la ansiedad. Es también posible que los sistemas de juego impuestos actualmente en el fútbol infantil en los que priman las rotaciones del total de miembros del equipo influyan en el bajo peso de la AIG-T sobre la ansiedad al sentirse todos los integrantes atraídos hacia su deporte, aunque este aspecto debe ser comprobado empíricamente. 
Continuando con la dimensión social de la cohesión, se formularon dos hipótesis. La primera de ellas postulaba la inexistencia de correlación entre el factor IG-S y el estado de ansiedad competitiva. Esta hipótesis se acepta, ya que no se halla correlación significativa entre este factor de la cohesión y las dimensiones de la ansiedad, unas observaciones que se ven respaldadas por los resultados hallados en otros estudios (Oh, 2014). La última hipótesis planteada proponía la inexistencia de influencia del factor AIG-S sobre las tres dimensiones de la ansiedad. Sin embargo, en contra de lo esperado, los análisis de revelaron la existencia de una correlación inversa estadísticamente significativa entre este factor de la cohesión y la dimensión preocupación de la ansiedad. Aunque estos resultados se contraponen a los encontrados en investigaciones anteriores (Prapavessis y Carron, 1996; Oh, 2014), sí se encuentran en concordancia con otros trabajos (Wolf et al., 2015). Esto puede deberse a que estos estudios, excepto la llevada a cabo por Oh y Gill (2014), realizan la investigación dentro del ámbito profesional o semiprofesional, en el que tanto los objetivos como la motivación de la competición son diferentes en comparación con el ámbito lúdico. Por otra parte, la investigación de Oh y Gill (2014) utiliza una muestra de deportistas en el ámbito recreativo y no profesional, sin embargo, esta cuenta con una población de sujetos adultos y obtiene resultados similares a los estudios anteriores. Por consecuente, la diferencia en los resultados encontrados puede hallarse en la edad de los sujetos. Por lo tanto, podría deducirse que, al tratarse de niños y al ser un ámbito deportivo en el que el objetivo principal es meramente lúdico, se dé más importancia a la dimensión social de la cohesión en comparación con jugadores adultos o que se dedican al deporte profesional.

Un último aspecto que destacar es que las relaciones entre la cohesión grupal y el estado de ansiedad competitiva observadas solo se dan sobre la variable preocupación, no hallándose influencia sobre ninguno de los dos factores restantes: ansiedad somática y desconcentración. El estudio de los estadísticos descriptivos revela que, dentro de la ansiedad, la variable con una media más alta es la preocupación, mientras que la ansiedad somática y la desconcentración puntúan una medias muy bajas y parecidas entre sí. En el artículo de adaptación y validación de la versión española de la Escala de Ansiedad Competitiva SAS-2 para deportistas de iniciación (Ramis et al, 2010) se observaron resultados similares, hallando puntuaciones altas en la escala de preocupación y puntuaciones bajas en las escalas de ansiedad somática y desconcentración. Esta concordancia resulta coherente ya que en ambos estudios se evalúan a futbolistas jóvenes, con una edad media similar, que se dedican al futbol de forma lúdica. Por otra parte, el hecho de encontrar una mayor puntuación media en la variable preocupación puede ser debido a otros factores, aparte de la cohesión grupal, que anteceden a esta variable como por ejemplo la presión ejercida por los adultos -padres y entrenadores- sobre los jugadores (Bohórquez et al, 2012). Para mas ende, al realizar un análisis descriptivo detallado sobre los ítems que componen el factor preocupación, se observa que el ítem con mayor puntuación media entre todos los sujetos es el número 5, el cual corresponde con la afirmación “... me preocupa desilusionar a los demás (compañeros, entrenadores, padres, etc)". Además, algunos estudios defienden que esta influencia sobre los jugadores es especialmente acusada en la primera infancia (Bohórquez y Checa, 2017). 
En relación con la baja puntuación en desconcentración que aparece en el estudio, una de las razones que puede explicar este hecho es que el deporte que se analiza en esta investigación, el fútbol, es un deporte grupal y no individual. Ramis et al. (2013), llevaron a cabo una revisión en la que hallaron mayores niveles de desconcentración en aquellos deportistas que se dedicaban a deportes colectivos en comparación con aquellos que jugaban en deportes individuales. Esto puede estar relacionado con que en los deportes individuales toda la responsabilidad del juego depende únicamente del deportista que compite lo que exige una mayor concentración, a diferencia de los deportes colectivos en los que la responsabilidad recae sobre un grupo de jugadores. Por consiguiente, la exigencia de concentración de un jugador que es miembro de un equipo es menor en comparación con la requerida en deportes individuales (Ramis et al., 2013).

Por último, la presente investigación cuenta con varias limitaciones. En primer lugar, en algunas categorías de la muestra analizada no se ha evaluado al equipo completo, sino a parte de él, lo cual podría ocasionar la pérdida de información sobre las distintas variables que se evalúan. Por otra parte, no se han tenido en cuenta ciertas variables que han podido influir en los resultados, como la influencia de los padres y entrenadores sobre los jugadores, algo que se podría abordar en futuras investigaciones. De igual modo, sería interesante estudiar la correlación existente entre los propios factores de la cohesión, ya que esto puede afectar a la influencia de estos factores sobre la ansiedad. Que la muestra estuviera compuesta exclusivamente por jugadores varones de fútbol limita el impacto de los resultados a este deporte y sexo concretos. Por último, resultaría de gran interés indagar en la relevancia del aspecto social de la cohesión en deportistas infantiles en comparación con deportistas adultos.

Como conclusión, cabe destacar que la cohesión grupal correlaciona inversamente con la ansiedad competitiva de los futbolistas de iniciación. Este resultado subraya, por tanto, la importancia de que los entrenadores intervengan sobre esta variable, lo cual permitirá la disminución de la preocupación vivida por los jugadores durante la competición que, a su vez, se verá reflejado en una mejora de su rendimiento deportivo.

\section{Referencias}

Aguinaga, I.; Herrero-Fernández, D.; y Santamaría, T. (2020). Factor protector de las estrategias de afrontamiento y la cohesión de grupo sobre el bienestar psicológico ante situaciones de ansiedad competitiva en futbolistas. Cuadernos de psicología del deporte, 21(1), 86-101.

Alan, Ü. y Kabasal, K. A. (2020). Effect of number of responde options on the psychometric properties of Likert-type scales used with children. Studies in Educational Evaluation, 66, 100895. https://doi.org/10.1016/j.stueduc.2020.100895

Barrasa, A.; y Gil, F. (2004). Un programa informático para el cálculo y la repsentación de los índices y valores sociométricos. Psicothema, 16(2), 329-335.

Bohórquez, M. R.; y Esquiva, I. (2017). Psychosocial factors related to the competitive anxiety of sports in training stages. Cultura, Ciencia y Deporte, 36, 205-210. https://doi.org/10.12800/ccd.v12i36.951

Bohórquez, M. R., Lorenzo, M., Bueno, M. R., y Garrido, M. Á. (2012). Influencia de la identidad grupal en la cohesión: Estudio piloto. Cuadernos de Psicología Del Deporte, 12(1), 151-154. https://doi.org/10.4321/S1578-84232012000100016 
Bohórquez, M. R., Vega, P., y Delgado, J. (2017). Rendimientos deportivos auto y heteropercibidos y cohesión grupal: un estudio exploratorio. Retos: Nuevas Tendencias de Educación Física, Deporte y Recreación., 31.

Borrego, C. C., Cid, L., y Silva, C. (2012). Relationship between group cohesion and anxiety in soccer. Journal of Human Kinetics, 34(1), 119-127. https://doi.org/10.2478/v10078-012-0071-z

Carron, A. V. (1982) Cohesiveness in sports groups. Interpretations and considerations. Journal of Sport Psychology, 4, $123-138$.

Carron, A. V., Shapcott, K. y Burke, S. (2007). Group cohesion in sport and exercise: past, present and future. En M. Beauchamp y M. Eys (Eds.), Group dynamics in exercise and sport psychology (pp. 117-141). Routledge.

Carron. A. V., Widmeyer. W. N. y Brawley L. R. (1985). The development of an instrument to assess cohesion in sport teams: The Group Environment Questionnaire. Journal of Sport Psychology, 7, 244-266.

Carron. A. V., Widmeyer. W. N. y Brawley L. R. (1988).Group cohesion and individual adherence to physical activity. Journal of sport and exercise psychology, 1022), 127-138.

Chambers, C. T.; y Johnston, C. (2002). Developmental Differences in Children's Use of Rating Scales. Journal of pediatric psychology, 271), 27-36. https://doi.org/10.1093/jpepsy/27.1.27

Chicau Borrego, C., Monteiro, D., Benson, A. J., Miguel, M., Teixeira, E., y Silva, C. (2021). Disentangling the effects of ego and taskinvolving climate perceptions on cohesion in youth sport. Sport, Exercise, and Performance Psychology. Advance online publication. https://doi.org/10.1037/spy0000270

Coleman, T., Godfrey, M., López-Gajardo, M. A., Leo, F. M., y Eys, M. (2021). Do it for the team: Youth perceptions of cohesion and role commitment in interdependent sport. Sport, Exercise, and Performance Psychology, 1022), 224-239. https://doi. org/10.1037/spy0000253

Eys, M., Hardy, J., Carron, A., y Beauchamp, M. (2003). The Relationship between Task Cohesion and Competitive State Anxiety. Journal of Sport and Exercise Psychology, 25, 66-76. https://doi.org/10.1123/jsep.25.1.66

Eys, M., y Kim, J. Team Building and Group Cohesion in the Context of Sport and Performance Psychology. Oxford Research Encyclopedia of Psychology. Retrieved 16 Jul. 2021, from https://oxfordre.com/psychology/view/10.1093/acrefore/9780190236557.001.0001/acrefore-9780190236557-e-186.

Golembiewski, R. T. (1962). The small group: an analysis of research concepts and operations. University of Chicago Press.

Grossbard, J. R., Smith, R. E., Smoll, F. L., y Cumming, S. P. (2009). Competitive anxiety in young athletes: Differentiating somatic anxiety, worry, and concentration disruption. Anxiety, Stress and Coping, 22(2), 153-166. https://doi. org/10.1080/10615800802020643

IBM Corp. Released 2017. IBM SPSS Statistics for Windows, Version 25.0. Armonk, NY: IBM Corp.

Leo, F. M., González-Ponce, I., Sánchez-Oliva, D., Pulido, J. J., y García-Calvo, T. (2015). Adaptation and validation in Spanish of the group environment questionnaire (GEQ) with professional football players. Psicothema, 27(3), 261-268. https://doi. org/10.7334/psicothema2014.247

Lott, A. J.; y Lott, B. E. (1965). Group cohesiveness as interpersonal atraction: a review of relationships with antecedent and consequent variables. Psychological Bulletin, 64(4), 259-309. https://doi.org/10.1037/h0022386

Martens, R., Vealey, R. y Burton, D. (1990) Competitive Anxiety in Sport. Human Kinetics.

Morris, L., Davis, D. y Hutchings, C. (1981). Cognitive and emotionals components of anxiety: Literature review and a revised worryemotionality scale. Journal of Educational Psychology, 73(4), 541-555. https://doi.org/10.1037/0022-0663.73.4.541

Nascimento Junior, J. R. A., Balbim, G. M., Vissoci, J. R. N., Moreira, C. R., Passos, P. C. B., y Vieira, L. F. (2016). Análise das Relações entre Ansiedade-Estado e Coesão de Atletas de Handebol. Psicologia - Teoria e Prática, 18(2), 89-102. https://doi. org/10.15348/1980-6906/psicologia.v18n2p89-102

Neil, R., Wilson, K., Mellalieu, S. D., Hanton, S., y Taylor, J. (2012). Competitive anxiety intensity and interpretation: A two-study investigation into their relationship with performance. International Journal of Sport and Exercise Psychology, 1022), 96-111. https://doi.org/10.1080/1612197X.2012.645134

Oh, E., y Gill, D. (2017). An Examination of the Relationship between Team Cohesion and Individual Anxiety among Recreational Soccer Players. Journal of Amateur Sport, 3(2), 1-26. https://doi.org/10.17161/jas.v3i2.5883

Prapavessis, H., \& Carron, A. V. (1996). The Effect of Group Cohesion on Competitive State Anxiety. Journal of Sport and Exercise Psychology, 18(1), 64-74. https://doi.org/10.1123/jsep.18.1.64 
Ramis, Y., Torregrosa, M., y Cruz, J. (2013). Revisitando a Simon \& Martens: la ansiedad competitiva en deportes de iniciación. Revista de psicologia del deporte, 22(1), 77-83.

Ramis, Y., Torregrosa, M., Viladrich, C., y Cruz, J. (2010). Adaptación y validación de la versión española de la Escala de Ansiedad Competitiva SAS-2 para deportistas de iniciación. Psicothema, 22(4), 1004-1009.

Ramis, Y., Viladrich, C., Sousa, C. y Jannes, C. (2015). Exploración de la estructura factorial de la Escala de Ansiedad Competitiva SAS2: Invariancia a través de lenguaje, género, edad y tipo de deporte. Psicothema, 27(2), 174-181. https://doi.org/10.7334/ psicothema2014.263.

Tenenbaum, G., Eklund, R., y Kamata, A. (2012). Measurement in Sport and Exercise Psychology. Human Kinetics.

Terry, P. C.; Carron, A. V.; Pink, M. J.; Lane, A. M.; Jones, G. J. W.; y Hall, M. P. (2000). Perceptions of group cohesion and mood in sport teams. Group dynamics: theory, research and practice, 4(3), 244.

Williams, J. M. (1991) Psicología aplicada al deporte. Biblioteca Nueva.

Wolf, S. A., Eys, M. A., y Kleinert, J. (2015). Predictors of the precompetitive anxiety response: Relative impact and prospects for anxiety regulation. International Journal of Sport and Exercise Psychology, 13(4), 344-358. https://doi.org/10.1080/161 2197X.2014.982676 